\title{
Rôle du développement agricole dans le développement économique dans les pays en voie de développement : Cas du Congo Brazzaville
}

\author{
Akouango Fulbert et \\ Kouako Jean Marie, \\ Université Marien Ngouabi
}

\section{Introduction}

Le développement économique est le processus par lequel la société peut utiliser efficacement les ressources économiques disponibles pour la production des biens et services nécessaires ${ }^{1}$. En fin de compte, cela mène à l'augmentation de ces produits et services pour la société et des individus. Par conséquent, la politique agricole inclut les moyens et méthodes utilisés par le Gouvernement pour orienter les ressources économiques vers la réalisation des objectifs sociaux et économiques désirés par la société.

Il est donc impératif d'étudier le développement économique et ses obstacles afin de pouvoir identifier les politiques agricoles appropriées qui doivent être adoptées en vue de la réalisation qui permettra d'atteindre les objectifs désirés.

Le développement économique peut être défini comme le processus au moyen duquel le revenu réel de la société peut être augmenté durant une logue période de temps aidant ainsi à fournir de plus grandes quantités de produits et de meilleurs services qui élèveraient le niveau de vie de l'ensemble de la société et des individus dans cette société ${ }^{2}$.

Par conséquent, nous pouvons dire que le principal objectif du développement économique est d'élever le niveau de vie de la majorité de la population. On utilise le revenu réel de préférence au revenu en espèces pour mesurer le taux de croissance du développement économique. Cela s'explique par le fait que le revenu réel est mesuré par rapport aux prix fixés et n'est pas affecté par la hausse générale des prix comme le revenu en espèces.

\section{Quelques considérations importantes}

L'augmentation du revenu réel est considérée comme une condition sine qua non pour élever le niveau de vie de la population et réaliser le développement économique. Cependant ce n'est pas la seule condition. Le Congo peut réaliser une augmentation du revenu réel mais échoue à réaliser le développement économique dans son sens le plus large.

Ce dernier peut être jugé sur la base des critères suivants :

\section{Distribution du revenu}

Le revenu national peut augmenter dans une période spécifique de temps, mais la distribution de ce revenu peut empirer, c'est-à-dire que le riche devient plus riche et que le pauvre devient plus pauvre.

Dans ce cas, le développement et le niveau de vie élevé sont confinés aux classes riches qui constituent un très bas pourcentage de la population. Par conséquent toute société doit garantir une distribution égale du revenu.

\section{Structure de la production}

L'augmentation de la production Congolaise peut être représentée par l'augmentation de la production d'équipement ou biens de capital, mais elle peut être 
accompagnée d'une réduction des biens de consommation qui sont considérés comme le déterminant majeur du niveau de vie de la population.

\section{L'utilisation des ressources économiques}

L'augmentation de la production et du revenu national peuvent considérablement dépendre des ressources naturelles ou de mauvaises conditions de travail, comme de longues heures de travail ou de mauvaises conditions personnelles, ou bien peuvent être réalisées aux dépens de la sécurité du temps de loisir ou de rapports personnels détériorés.

\section{Caractéristiques générales du Congo}

\section{La production primaire}

Les matières premières agricoles et non agricoles, sont considérées comme les produits les plus importants du Congo. Cela est attribué à la disponibilité de certains intrants de production comme le sol, la main-d'œuvre, et aux moyens d'industrialisation peu avancés du pays. Le pourcentage élevé de la main-d'œuvre travaillant dans le secteur agricole (75 - 90\%), en plus du fait que le revenu agricole constitue à peu près $50 \%$ du revenu national, indique que la production est concentrée dans les matières premières, en particulier le pétrole. Dans la plupart des cas, la production nationale est restreinte à deux ou trois produits. Au Congo, les principales caractéristiques de la production primaire sont le manque d'utilisation optimale des ressources économiques de la production, en résultat du capital réduit des petites parcelles de terre agricole cultivées par les individus, de la basse fertilité du sol de la main-d'œuvre peu efficace et des compétences techniques médiocres ainsi que de l'inefficacité des organismes et des agences liés à la production et à la commercialisation.

\section{Pression de la population}

Les pressions de la population prennent trois formes différentes :

- La propagation du chômage dans le secteur agricole ;

- Le taux de natalité élevé qui entraîne le fait qu'un grand pourcentage du nombre des individus dépend d'autres personnes pour leur substance ;
- Le taux de mortalité réduit et le taux de natalité élevé qui mène à un taux élevé de croissance de la population diffère considérablement entre pays avancés et le Congo.

Au Congo à peu près $50 \%$ de la population est âgée de moins de 20 ans. D'autre part, l'espérance de vie est bien inférieure que dans les pays avancés (40 45) comparé à (65 - 70) dans les pays avancés.

\section{Retard}

Le retard signifie le bas niveau de culture et de santé de la population ou particulier relativement aux salariés du secteur primaire entraînant la diminution de l'utilisation optimale des éléments de production. Cela est attribué au taux élevé d'analphabétisme, de malnutrition, de mauvaises conditions sanitaires, de manque de formation, d'obstacles variés entravant le mouvement de la main-d'œuvre d'une profession à l'autre, outre la présence de certaines traditions liées aux systèmes familiaux et tribaux. Par conséquent, le Congo souffre d'une pénurie aiguë d'innovations qui pourraient développer des moyens de production et de distribution.

L'augmentation de la production et du revenu national peuvent considérablement dépendre des ressources naturelles ou de mauvaises conditions de travail.

Sous - développement

Il est attribué à la rareté d'utilisation des moyens technologiques modernes en matière de production, sans compter l'absence de systèmes socioéconomiques développés. Le Congo a des ressources naturelles et agricoles suffisantes qui sont soit inutilisées, soit utilisées de façon inefficiente, entraînant l'usage inefficace des facteurs de production.

\section{Pénurie du capital}

Le Congo souffre d'une grande pénurie de ressources en capital entraînant ainsi la réduction de l'investissement dans la production.

Ce capital est estimé à $5-10 \%$ de celui des pays avancés. Cela est attribué à la réduction des investissements annuels à 5 - 7\% comparé à $15-20 \%$ 
dans les pays avancés. Étant donné que le revenu par habitant est très bas, par conséquent, $95 \%$ de ce revenu est dirigé vers la consommation laissant seulement $5 \%$ pour l'épargne et l'investissement ;un pourcentage qui ne suffit absolument pas pour de grands projets d'investissements. On remarque également que ce pourcentage d'épargne dérisoire est dirigé vers l'investissement à court terme de préférence à l'investissement à long terme.

\section{Orientation vers l'investissement étranger}

L'économie du Congo dépend du commerce étranger qui est basé sur l'exportation des produits de base.

En résultat, le pays est exposé à de grands bouleversements dans son activité économique. De plus, la concentration sur la production orientée vers l'exportation fait lourdement dépendre l'investissement sur les prêts étrangers, principalement orientés vers le développement de l'industrialisation des produits de base, déséquilibrant ainsi le développement de l'économie. D'autre part, le Congo dépend de l'importation pour satisfaire sa demande en produits alimentaires et articles manufacturés créant un grand écart entre la valeur des exportations et celle des importations, d'où le déficit continuel de la balance de paiement.

\section{Les bouleversements dans les prix des produits de base exportés par le Congo sont bien plus élevés que ceux des articles manufacturés exportés par les pays avancés.}

\section{Obstacles au développement}

Les obstacles les plus importants qui confrontent le développement sont les suivants.

\section{Imperfections du marché}

Les imperfections du marché comprennent tous les aspects déjà mentionnés aux sous-titres sousdéveloppement et retard, à savoir la difficulté du mouvement des facteurs de production entre les différents usages, le manque de flexibilité des prix, l'ignorance des conditions et du potentiel de la commercialisation, la structure sociale fixe, qui gênent l'utilisation optimale des ressources optimales.

Nous remarquons aussi l'efficacité d'exploitation productrice des ressources extrêmement réduites, entravant la réalisation en temps opportun du développement désiré.

Au cours des dernières années le pays a mis en œuvre des programmes de réforme économique et d'ajustement structural. Cependant, certains de ces programmes n'ont pas eu le succès désiré étant donné le manque d'efficacité des marchés du pays.

\section{Cercles vicieux}

Les cercles vicieux sont constitués par un groupe de variables économiques étroitement liées en forme de boucle. Cela signifie que chaque variable est le résultat de la précédente et la cause de la suivante, de sorte qu'en commençant à un point de la boucle nous finissons par y revenir. La diminution du revenu réel est attribuée à celle de l'utilisation optimale des facteurs de production, entraînant une réduction de l'épargne et, par conséquent, de l'investissement. Le retard des ressources humaines entraîne celui des ressources naturelles.

En même temps, la diminution du revenu réel entraîne la réduction de la demande, donc de l'investissement. Ainsi donc, la corrélation de ces variables dans les cercles vicieux susmentionnés perpétue la pauvreté du pays, c'est-à-dire que "le pays est pauvre parce qu'il est pauvre".

\section{Forces internationales}

De nombreuses études économiques soulignent le fait que les avantages et les bénéfices du commerce étranger sont recueillis par les pays avancés qui exploitent le Congo où ils puisent les matières premières nécessaires pour leur industrie et commercialisent leurs produits. Les bouleversements dans les prix des produits de base exportés par le Congo sont bien plus élevés que ceux des articles manufacturés exportés par les pays avancés. Cela influence grandement le revenu national et la balance de paiement du Congo. 


\section{Taux de croissance élevé de la population}

Le Congo affronte un taux élevé de croissance de la population qui peut absorber tous les aspects du développement économique réalisé par d'autres facteurs. Dans certains cas, le taux élevé de croissance de la population est considéré comme l'un des obstacles les plus sérieux confrontant le développement.

Le taux de croissance élevé de la population exige le taux de consommation élevé et entraîne la réduction de l'épargne et de l'investissement.

Ces obstacles à la croissance économique sont corrélatifs et complexes. Par conséquent, l'intervention du Gouvernement de la république est impératif afin d'élaborer la politique qui romprait la corrélation entre les différents facteurs et variables et offrirait le climat approprié pour le lancement de la croissance économique. Le Gouvernement peut adopter une politique dans le but de briser le cercle vicieux, afin d'amener le développement désiré. On peut commencer par le point le plus faible à savoir, entre l'épargne et l'investissement.

En vue d'encourager ces dernières, le Gouvernement peut changer la politique fiscale ou monétaire. Il peut aussi, dans ce sens, obtenir des emprunts étrangers ou des subventions des pays donateurs ou d'institutions financières internationales.

\section{Politique agricole}

Toute politique est concernée par l'usage de certains instruments par l'intermédiaire desquels elle intervient dans l'activité économique, afin de changer le modèle d'utilisation des ressources économiques. Ces instruments sont appelés mécanismes de politique. Étant donné que dans certains cas les marchés ne réalisent pas l'objectif désiré du développement économique, par conséquent, le système efficace exige la présence d'institutions gouvernementales d'une grande compétence, un cadre régulateur approprié, un système de droit de propriété clair et spécifique et un système juridique solide.

Bref tout gouvernement peut jouer un rôle majeur dans l'utilisation du développement économique par l'élaboration de la politique visant au développement et à l'augmentation de l'efficacité des procédés de production et de commercialisation.

La figure 1 donne quelques exemples de politiques qui peuvent être utilisées pour éliminer les obstacles qui confrontent la production et la commercialisation et qui constituent des incitations majeures pour le développement économique.

Figure 1

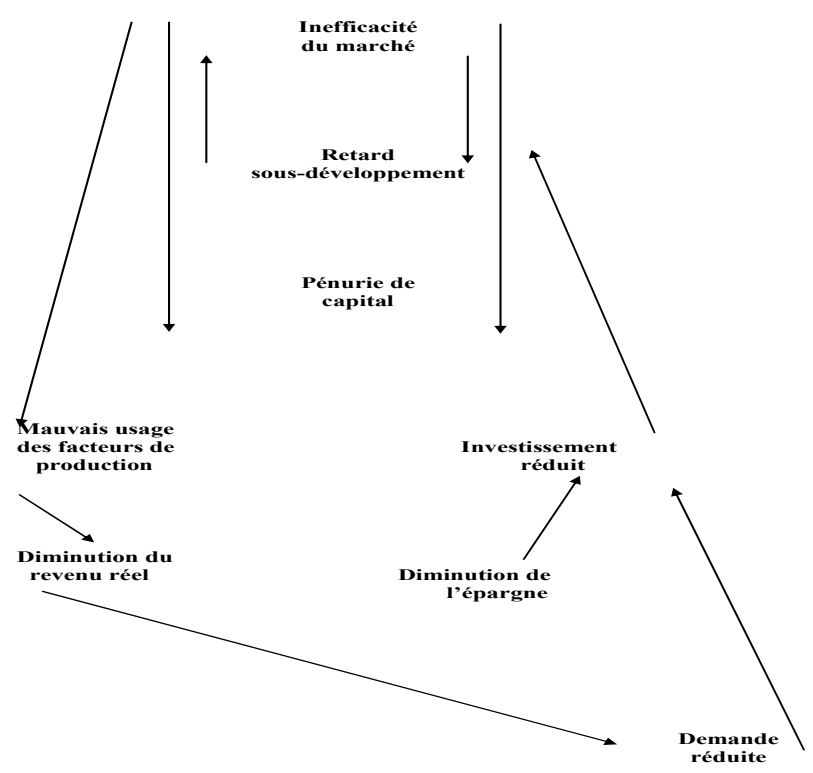




\section{Cercles vicieux de la pauvreté}

Investissement dans l'infrastructure c'est-à-dire les routes, les moyens de transport, l'énergie, l'infrastructure des moyens de production, à savoir, les systèmes d'irrigation et de drainage, le développement des ressources humaines dans les domaines de l'enseignement et de la santé, de même que l'investissement dans la recherche et le développement technologique. Ces politiques doivent encourager et promouvoir l'intégration internationale aux fins d'un système économique stable.

- Politique relative au commerce et au taux de change étrangers.

- Politique encourageant le secteur public et privé à investir dans le développement des services de production et de commercialisation.

- Politique monétaire judicieuse qui permet l'augmentation du potentiel de crédit pour le secteur privé.

- Politique relative au développement du système des lois et des institutions relatives à la production et la commercialisation.

Par conséquent, les instruments de la politique agricole peuvent être divisés comme suit : la politique basée sur les prix, la politique non basée sur les prix, la politique macroéconomique, sectorielle ou fondée sur les produits.

\section{Politique basée sur les prix}

Elle comprend la subvention sur l'impôt et les prix des produits et des intrants de production, comme la subvention sur le taux d'intérêt, les semences ou les engrais chimiques. Elle comprend également l'impôt sur la main-d'œuvre ou la terre agricole, l'ajustement du taux de change, en plus du changement du niveau général des prix au moyen de la politique monétaire ou pécuniaire qui a un impact direct sur le taux d'inflation.

\section{Politique non basée sur les prix}

Elle comprend l'intervention du gouvernement qui ne vise pas directement à changer les prix. La politique non basée sur les prix est utilisée afin de déterminer les quantités, non les prix comme la détermination des quantités importées d'un certain produit au lieu du changement des droits de douane qui influence les prix des produits importés. Le gouvernement peut aussi mettre des limites aux opérations de crédit et de prêt, comme alternative à la hausse du taux d'intérêt.

\section{La politique qu'elle soit ou ne soit pas basée sur les prix est à trois niveaux}

Quelques exemples de la politique non basée sur les prix comprend l'investissement dans la recherche scientifique, la redistribution des investissements publics et des dépenses courantes sur les diverses activités économiques, le développement et l'amélioration des méthodes de distribution des moyens de production et des prêts, l'encouragement à des technologies modernes, l'imposition de restrictions commerciales quantitatives et l'adoption de la politique démographique.

La politique qu'elle soit ou ne soit pas basée sur les prix est à trois niveaux ${ }^{3}$ :

1 - Politique au niveau du produit ou de l'élément de production. Elle vise au changement du rapport entre les éléments de production et les produits finis.

2 - Politique au niveau sectoriel, visant au transfert $\mathrm{du}$ revenu agricole au secteur industriel ou de consommation. Dans ce cas le taux d'échange commercial n'est pas favorable au secteur agricole. Citons en exemple la politique basée sur les prix, le taux de change et les droits de douane visant à la protection de la production locale un exemple de la politique non basée sur les prix est la restriction quantitative imposée aux importations.

3 - Politique au niveau national, visant au changement du niveau général des prix. Elle comprend la politique relative au taux de change des devises étrangères, au commerce, à l'impôt et aux subventions. À ce niveau, la politique non basée sur les prix inclue les dépenses gouvernementales et les changements de l'offre en espèces. Il est très important de souligner que le niveau général des prix joue un rôle important dans l'identification de la compétitivité des produits de l'état sur les marchés internationaux. Il identifie également le taux d'échange 
commercial entre les producteurs et les consommateurs et entre les débiteurs et les créanciers, en plus de son impact sur le budget public de l'état.

\section{La détermination des prix et le problème économique}

Les sociétés primitives ne souffraient pas de problèmes économiques car les produits et les services disponibles étaient en excès de la demande. Par conséquent, chaque individu dans la société satisfait ses besoins en produits et services sans entrer en conflit avec les besoins des autres. Mais avec le nombre croissant de la population, les quantités de produits et de services sont devenues inférieures aux besoins créant ainsi un conflit quant à la satisfaction des besoins des individus ${ }^{4}$. Tel est le problème économique aigu dont souffre le monde. L'insuffisance des produits et des services existants et les besoins croissants ont incité les responsables dans des nombreux pays à chercher un moyen pour distribuer les produits et les services disponibles de façon à satisfaire les différents besoins 5 .

Les sociétés primitives ne souffraient pas de problèmes économiques car les produits et les services disponibles étaient en excès de la demande.

Les systèmes en conséquence diffèrent quant à leurs moyens de traiter les problèmes économiques. Le système socialiste suivi par le bloc des pays de l'Est sous l'ex-Union Soviétique a adopté la planification économique centralisée ${ }^{6}$. Dans ce système, toutes les ressources économiques de la société étaient identifiées, ensuite on dressait une liste des produits et services à offrir, enfin on détermine la méthode de production et de distribution. Cela signifie que les décisions de production et de distribution étaient prises au gré de comités de planification dans tous les pays et à des niveaux variés. Ni le marché, ni le prix ne jouaient un rôle dans l'affrontement du problème économique (à l'exception de ce qui se passait au marché noir). Quant au système capitaliste suivi par le bloc occidental sous la direction des États-Unis d'Amérique, il dépendait principalement des mécanismes du marché et de l'incitation au profit pour résoudre le problème économique. La rentabilité était et demeure le principal stimulant de toute activité économique de ce système. Étant donné que la rentabilité dépend de la différence entre les revenus et les coûts de toute activité économique et que les prix sont considérés comme les principaux déterminants des coûts et des revenus, le marché et les prix, par conséquent, sont devenus le déterminant des quantités de denrées et de services produits de même que les méthodes de production et de distribution.

Cela signifie que les marchés et les prix aident à résoudre le problème économique en prenant les décisions suivantes ${ }^{7}$ :

- quantité de produit et de services

- méthode et coûts de production

- forme, moment et lieu de distribution de ces ressources

- individus à qui ces produits seront distribués et quota de chaque individu.

Les mécanismes du marché, c'est-à-dire l'offre et la demande, déterminent la réponse à ces questions. Il faut d'abord comprendre les principes et la nature de ces mécanismes, afin de comprendre le coût dans la fixation des prix.

\section{Offre}

La courbe de l'offre d'un produit donné est définie comme les quantités variées qui peuvent être produites et offertes à différents niveaux de prix. L'offre peut être estimée au moyen de l'information obtenue des unités produisant ce produit ${ }^{8}$. Les personnes pour qui la théorie économique est familière se rappellent que la courbe de l'offre du produit est la courbe des coûts marginaux de ce produit, tandis que la courbe totale de l'offre sur le marché est totalement horizontale des courbes de l'offre pour tous les producteurs de cet article. Étant donné que l'on prévoit que les coûts marginaux augmenteront de la production, il est également prévisible que la courbe de l'offre totale sera positive c'est-à-dire que la production augmentera à mesure qu'il y a un rapport direct entre la quantité et le prix sur la courbe de l'offre.

\section{Demande}

La courbe de la demande d'un produit est définie comme les quantités variées que les consommateurs désirent et sont capables d'acheter à différents ni- 
veaux de prix. La courbe de la demande dépend considérablement des désirs des consommateurs et de leurs niveaux de revenus ${ }^{9}$. Plusieurs raisons logiques donnent à la courbe une direction négative. Cela signifie qu'il y a un rapport négatif entre le prix et la quantité demandée.

L'offre et la demande interagissent pour déterminer le prix de marché d'un produit donné. Le prix d'équilibre du produit est au point d'intersection de l'offre et la demande ${ }^{10}$. Par conséquent, le prix d'équilibre est celui qui rend égales les quantités offertes et celles qui sont demandées. Dans ce cas, la quantité de produits offerts et, en même temps, les besoins du marché sont satisfaits.

Il arrive parfois que les prix du marché soient différents du prix d'équilibre. Dans ce cas, le marché n'est plus équilibré, créant ce qu'on appelle un produit déséquilibré ou l'offre n'est pas égale à la demande à ce prix particulier. Si le prix est inférieur au prix d'équilibre, les quantités demandées (NS) sont (conformément à la courbe de la demande) supérieures aux quantités offertes (AS) (conformément à la courbe de l'offre) à ce prix. Cela signifie qu'il y a un surplus de demandes ainsi que l'indique la figure 2 c'est-à-dire que les demandes et désirs des consommateurs ne sont pas satisfaits. Cette situation crée une sorte de concurrence entre les consommateurs pour obtenir le produit entraînant spontanément la hausse de son prix, le rapprochant ainsi progressivement du prix d'équilibre. D'autre part, si au contraire le prix du marché est plus élevé que le prix d'équilibre, la quantité offerte sera plus grande que la quantité demandée c'est-à-dire qu'il y $a$ un excès d'offre.

Cela crée une concurrence entre les producteurs pour vendre leurs produits à des prix plus bas rapprochant ainsi progressivement le prix du marché du prix d'équilibre. Par conséquent, la concurrence du marché crée une sorte de correction spontanée des changements des prix et les fait revenir au niveau d'équilibre sans l'intervention du gouvernement Congolais. La présence d'un marché où la concurrence est libre garantit la détermination des prix de façon correcte et précise où les prix de production (tels qu'ils sont représentés par la courbe de l'offre) s'accorderont aux désirs et aux potentiels des consommateurs (tels que représentés par la courbe de la demande).

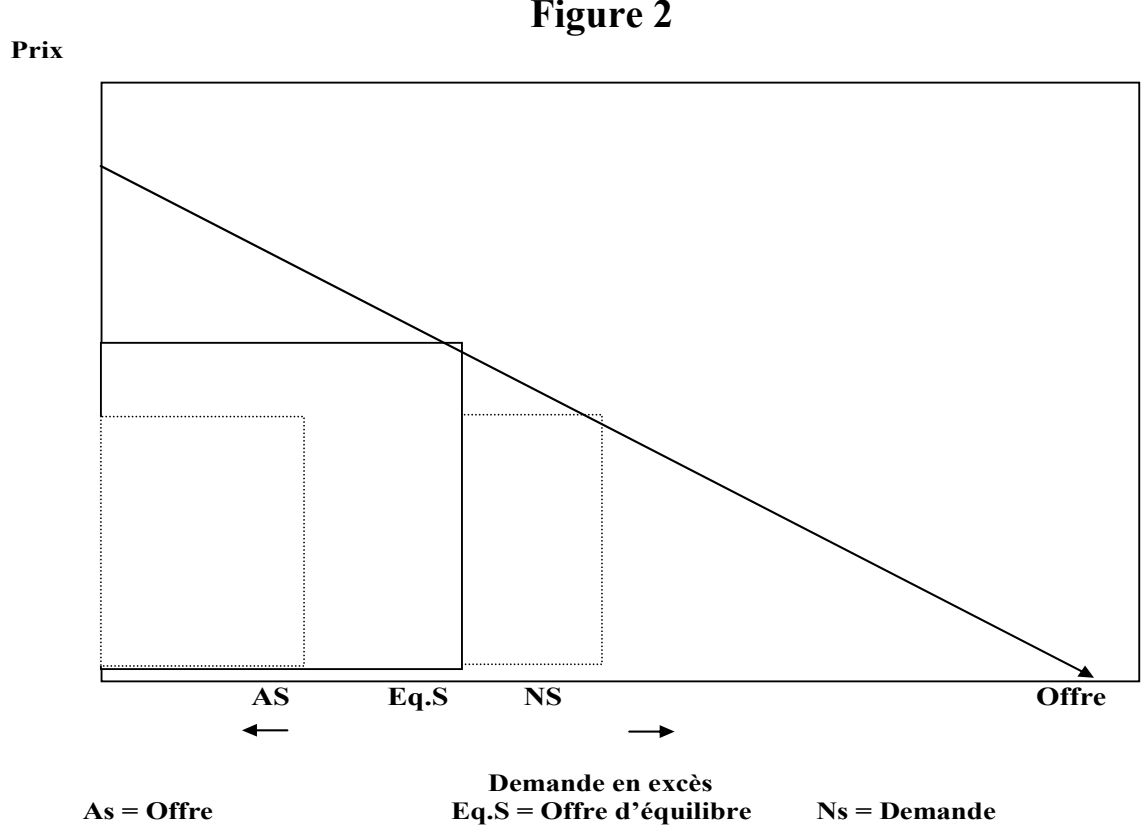

Changement de l'offre et de la demande

L'offre et la demande, et par conséquent le prix, étaient déterminées dans la figure 2 pourvu que les autres facteurs fussent constants ${ }^{11}$. Si ces facteurs changent, l'offre ou la demande, ou les deux, changeraient, et entraîneraient par conséquent, un changement de prix. 
Les facteurs suivants entraînent le changement de l'offre :

- le changement technologique entraîne l'augmentation ou la réduction de l'utilisation optimale des facteurs de production.

- le changement des prix des intrants. Les facteurs suivants entraînent le changement de l'offre :

- Changement dans les revenus des consommateurs

- Changement du nombre des consommateurs

- Changement des goûts et des habitudes des consommateurs

- Changement de prix des produits de substitution ou complémentaires

- Prévision des prix à venir.

Le changement de l'un de ces facteurs peut entraîner le changement de l'offre ou de la demande, ou des deux, entraînant, par conséquent, le changement du prix et de la quantité de l'équilibre. Étant donné que ces facteurs changent constamment, on peut prévoir que les prix d'équilibre changeront constamment.

\section{Structure du marché}

Les marchés diffèrent selon le degré de concurrence. Plusieurs facteurs déterminent ce degré et donc déterminent la spécificité du marché se sont 12 :

- le nombre de vendeurs et d'acheteurs

- le degré d'homogénéité du produit

- le degré de liberté dans les transactions (vente ou achat) du marché.

- la disponibilité des informations relatives à la commercialisation.

En nous basons sur ces facteurs, nous pouvons classifier les marchés dans les catégories suivantes :

\section{Marché de la concurrence parfaite}

On y trouve un grand nombre de vendeurs et d'acheteurs, de sorte que les transactions de chaque individu sont réduites afin qu'il ne contrôle, n'influence le prix. Le produit sur le marché doit être homogène du point de vue de l'acheteur pour que ce dernier ne préfère pas un certain vendeur et pour que le vendeur ne préfère pas un certain acheteur. Le marché ne doit pas comporter d'obstacles empêchant les individus, vendeurs ou acheteur, d'effectuer une transaction. De plus, chacun doit être parfaitement informé des aspects économiques et commerciaux du marché, comme les prix locaux courants et les prix escomptés, les prix internationaux courants et escomptés, les quantités faisant l'objet de contrats de commercialisation au niveau local ou international, la production prévue pour un article de commerce.

\section{Marché de concurrence monopolistique}

C'est le marché dont est absent la deuxième condition citée ci-dessus. Il y a un grand nombre de vendeurs et acheteurs, mais le produit n'est pas homogène du point de vue de l'acheteur. Le manque d'homogénéité peut être réel, comme une différence de qualité, ou bien cela peut être une différence provenant du triage du calibrage ou de l'emballage. Parfois la différence n'est pas réelle mais les méthodes de réclamation et de publicité convainquent l'acheteur que le produit est différent. Du point de vue économique, il est important que l'acheteur soit convaincu que le produit vendu par un certain vendeur est meilleur que le même produit vendu par les autres vendeurs. Cette différence permet au vendeur de vendre son produit à un prix plus élevé. Autrement dit, il y a des différences entre les prix du produit, dues à la différence et à l'avantage de ce produit chez les divers vendeurs.

\section{Marché oligopole}

Le nombre de vendeurs d'un certain produit est limité, de sorte qu'ils peuvent s'entendre entre eux pour l'adoption d'une politique de production déterminée (par l'augmentation où la réduction de la production), ou d'une certaine politique de détermination des prix (par exemple hausser ou baisser le prix).

\section{Marché complètement monopolistique}

On distingue un seul vendeur qui contrôle la quantité offerte ou le prix d'un produit. Bref, c'est le marché qui garantit l'utilisation optimale des ressources économiques disponibles ${ }^{13}$. Le gouvernement du 
Congo, qui depuis 1991, en effectuant le changement de la planification centralisée pour l'adoption de mécanismes du marché doit élaborer les lois et les législations et mettre en place la conjoncture qui garantit la prédomination complète (ou de la libre concurrence).

\section{Intervention du gouvernement dans la fixation des prix}

Le gouvernement suit certaines politiques de détermination des prix afin de s'éloigner du niveau géné- ral des prix d'équilibre et de réalisation des objectifs spécifiques qu'il vaut mieux ne pas laisser au gré des prix du marché. Citons en exemple les pays de la zone Euro qui fixent, pour un prix plus élevé que le prix d'équilibre dans le but d'encourager l'augmentation de la production et de maintenir des niveaux de revenus plus élevés pour les producteurs 14.

Dans le cas ces quantités sont exportées, l'union doit supporter le fardeau des subventions à l'exportation si le prix international est inférieur au prix qu'elle a fixé ainsi que l'illustre la figure 3

Prix

Figure 3

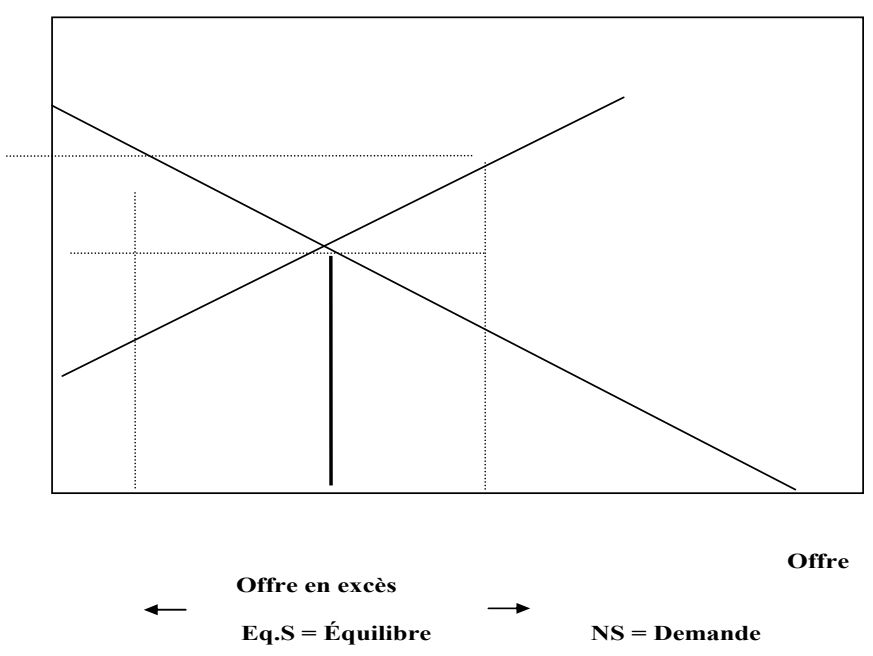

\section{Conclusion}

Le gouvernement congolais dans la lutte contre la pauvreté, fixe le prix au niveau inférieur au prix d'équilibre, ce qui entraîne la réduction de la production et l'augmentation des quantités demandées. Cela entraîne un excès de demande, ainsi que 1'illustrait la figure 2.Une fois les prix fixés, on assiste à la rareté du produit sur le marché.

Dans ce cas, le gouvernement est obligé d'importer ces quantités du produit demandé, ajoutant ainsi le fardeau considérable au budget de l'état.

Les produits agricoles sont incontestablement à la base du développement du Congo. Leur valorisation à travers une bonne politique de production et d'accès au crédit augmenterait les performances.

\section{Notes et références}

1. 3 - BERTHELEMY J.C et GIRARDINE., 1991 «Le surendettement et stratégies des PVD» In : Revue d'économie politique, 101 (4) Juillet - août, pp. $524-$ 604

5 - CHAMBAS et DIAW A., 1984. «Les facteurs explicatifs du comportement des états fournisseurs d'aide » communication au congrès international des économistes de langue Française, Clermont Ferrand 2426 Mai.

19 - J.F. COUET et J. BREMOND 1978, « pays sousdéveloppés ou pays en voie de développement, » Tome 1

2. 1 - ARTUSP, 1991. «Épargne mondiale : la pénurie ». IN : Revue option finance, 29 octobre 1990, produits dans problèmes économiques, $\mathrm{N}^{\circ} 2207,9$ janvier, $\mathrm{PP}$ $17-21$ 
4 - CASHIN P. et PATTILlOC, 2000 «la durée des chocs des termes de l'échange en Afrique subsaharienne » Finances et développement, juin pp 26-29

22 - MATHONAT J. 1984 « les effets du financement extérieur sur la structure de la dépense publique dans les pays en développement» communication au Congrès international des économistes de langue Française, Clermont Ferrand 24-26 Mai

3. 20 -LFEBER. L, 1979. "The role of aide two views ou raid and développement», Economic louncil of Canada, may, 278-286.

26 -PATRICK GUILLAUMONT. 1985 « Economie du développement » Tome 1, le sous-développement.

4. 21 -MATHONAT J. 1984. «Rôle respectif des politiques internes et des facteurs externes dans l'endettement des pays africains au Sud du Sahara » Communication à la conférence sur le redressement économique de l'Afrique au subsaharien, OCDE, Paris 14-16 Novembre.

24 - OLIVIER CORTES ET SEBASTIEN JEAN 1997. 3 Les échanges internationaux modifient la demande de travail » In Economie et statistique N³01-302, 1997 $1 / 2$.

5. 25 - ORLY J.N, 1994 « conversion - réduction de dette dans les pays en voie de développement (PVD) : bilan théorique et impacts macro-économiques » In revue d'économie financière, $227-351$.

18 - KESSLER D et ULMO P.A., 1985 «éléments pour déterminer le niveau optimal d'endettement d'un pays » In : Mondes en développement, Tome 13, N 50 / 51, pp 259 - 273.

6. 12 - GRIGNOLS E et BAGHWATI J, 1979. «Foreign capital, shavings and dépendance» a replay to MR WASSOW » In the review of economics' and statistics, vol $61 \mathrm{n}^{\circ}$, Février, pp. 10-21

13 - HERNANDEZ - CATA I, 2000 " croissance et investissement en Afrique subsaharienne : Que peut-on faire ? » In: Finances et développement, décembre, pp. 30-33.

16 - KESSLER D., «Endettement Épargne et croissance dans les pays en développement », In Épargne et développement, 1985, pp 383 - 395.
7. 8 - COHEN D, 1996 «La dette oubliée des pays en développement », In: Turbulence et spéculation dans l'économie mondiale, pp $293-208$.

14 - H.W. ARDT., 1987, « Développement économique » La marche d'une idée

15 -KESSLER D, 1984 « Les problèmes causés par la crise financière internationale et leurs répercutions sur la structure financière des économies en développement », communication du Congrès international des économistes de langue Française Clermont Ferrand 24 26 mai.

8. 9 - DJONDANG P, 1985 « Importations de capitaux et croissance économique », Economica, $175 \mathrm{p}$.

10 - EBOUE. C, 2000. « Le traitement de l'endettement extérieur » In : Formation en modélisation et macroéconomie appliquée, LOME 17 - 18 Avril, Ministère de la planification et du développement.

11 - GRIFFIN K. et ENOS J., 1970. « Foreign assistance : Objectives and conséquences $»$ In : Economic développement and cultural change April, 278 - 286.

9. 6 - CODERT. V. 1990 «Les disparités internationales de comportements d'épargne. », In : Economie et stratégie $\mathrm{N}^{\circ} 232$, mai, 52

7 - COHEN D, 1985. «La dette des nations », In : cahiers du CERNEA, $N^{\circ} 19$, septembre, PP 6-34.

10. 17 -KESSLER D ET Strauss - Kahn d, 1984, 3. Existe-t-il un lieu entre l'épargne intérieure et l'afflux de Capitaux extérieurs? », In: Revue Tiers Monde 1098, avril - juin, pp 269 - 298.

23 -OLIVEIRA - MARTIONS $\mathrm{J}$ et PLIHON D, $1990 »$ l'impact des transferts internationaux d'épargne sur les déséquilibres extérieurs » In Economie et Stratégiques $\mathrm{N}^{\circ} 232$, mai, 33 - 48.

11. CASHIN P. et PATTILLOC, 2000, op. cit.

12. 2 - AUVERNY - BENNETOT p., 1991. «la dette du tiers monde », la documentation Française, 126 p. BERTHELEMY J.C et GIRARDINE., 1991, op. cit.

13. ARTUSP, 1991. op. cit

14. CHAMBAS et DIAW A., 1984 op. cit. 